\title{
Adolescentes na Era Digital: Impactos na Saúde Mental
}

\author{
Adolescents in the Digital Age: Impacts on Mental Health \\ Adolescentes en la era digital: Impactos en la Salud Mental
}

Recebido: 01/07/2021 | Revisado: 07/07/2021 | Aceito: 12/07/2021 | Publicado: 23/07/2021

\author{
Synara Sepúlveda Sales \\ ORCID: https://orcid.org/0000-0002-7792-4952 \\ Universidade Tuiuti do Paraná, Brasil \\ E-mail: synarasepulveda@hotmail.com \\ Talita Mendes da Costa \\ ORCID: https://orcid.org/0000-0002-9536-4952 \\ Universidade Cesumar, Brasil \\ E-mail: talitamc15@gmail.com \\ Maria Julia Pegoraro Gai \\ ORCID: https://orcid.org/0000-0002-8481-1112 \\ Universidade Federal de Santa Catarina, Brasil \\ E-mail: mariajuliagai@hotmail.com
}

\begin{abstract}
Resumo
A expansão do uso da tecnologia tem interferido nos diversos aspectos da vida cotidiana das pessoas, em especial, os adolescentes. Mesmo com os benefícios dos avanços tecnológicos, o uso por parte dessa faixa etária sem a supervisão adequada e uso excessivo pode gerar diversos riscos e significativas alterações na saúde mental. Tendo isso em vista, este trabalho buscou identificar os impactos da exposição à internet e mídias sociais na saúde mental dos adolescentes. Foi realizada uma revisão integrativa com coleta de dados na Web of Science, Scopus, BVS e Psycnet. Do total de 245 artigos identificados, exclui-se os documentos de acordo com os critérios de inclusão e exclusão, restando 40 artigos para serem analisados. Como resultados, constatou-se a imprescindibilidade da tecnologia para a inserção do jovem neste mundo que evolui a cada dia. Entretanto, o uso desmedido dos recursos tecnológicos pode afetar os hábitos alimentares, sedentarismo, agressividade, uso ou abuso de substâncias, depressão, distorções da imagem corporal, ciclo sono/vigília, hiperatividade, automutilação e ideações suicidas. É inviável pensar nas novas gerações desvinculadas dos meios digitais, por esta razão observa-se a necessidade de uma atuação conjunta das escolas, pais, responsáveis e comunidade em geral para o desenvolvimento de ações que abordem de forma clara os riscos do mundo virtual.
\end{abstract}

Palavras-chave: Adolescente; Saúde mental; Digital; Internet.

\begin{abstract}
The expansion of the use of technology has interfered with different aspects of people's daily lives, especially teenagers. Even with the benefits of technological advances, the use by people of this age group without adequate and excessive use can generate several risks and relevant changes in mental health. With this in mind, this work sought to identify the impacts of exposure to the internet and social media on the mental health of adolescents. An integrative review was carried out with data collection from Web of Science, Scopus, BVS and Psycnet. Of the total of 245 articles identified, documents were excluded according to the inclusion and exclusion criteria, leaving 40 articles to be allowed. As a result, it was found that technology is essential for the insertion of young people in this world that evolves every day. However, the excessive use of technological resources can affect eating habits, sedentary lifestyle, aggressiveness, substance use or abuse, depression, body image distortions, sleep/wake cycle, hyperactivity, selfmutilation and suicidal ideations. It is unfeasible to think about the new generations disconnected from digital media, for this reason there is a need for a joint action of schools, parents, guardians and the community in general to develop actions that clearly address the risks of the virtual world.
\end{abstract}

Keywords: Adolescent; Mental health; Digital; Internet.

\section{Resumen}

La expansión del uso de la tecnología ha interferido en diferentes aspectos de la vida cotidiana de las personas, especialmente en los adolescentes. Incluso con los beneficios de los avances tecnológicos, el uso por parte de personas de este grupo de edad sin una adecuada supervisión y un uso excesivo puede generar varios riesgos y cambios significativos en la salud mental. Con esto en mente, este trabajo buscó identificar los impactos de la exposición a internet y las redes sociales en la salud mental de los adolescentes. Se realizó una revisión integradora con recolección de datos de Web of Science, Scopus, BVS y Psycnet. Del total de 245 artículos identificados, los documentos fueron excluidos según los criterios de inclusión y exclusión, quedando 40 artículos para analizar. Como resultado, se encontró que la tecnología es fundamental para la inserción de los jóvenes en este mundo que evoluciona a cada día. 
Sin embargo, el uso excesivo de recursos tecnológicos puede afectar la alimentacion, el sedentarismo, la agresividad, el uso o abuso de sustancias, la depresión, las distorsiones de la imagen corporal, el ciclo sueño/vigilia, la hiperactividad, la automutilación y las ideas suicidas. Es inviable pensar en nuevas generaciones desconectadas de los medios digitales, por ello es necesaria una acción conjunta de escuelas, padres, tutores y la comunidad en general para desarrollar acciones que aborden claramente los riesgos del mundo virtual.

Palabras clave: Adolescente; Salud mental; Digital; Internet.

\section{Introdução}

O uso de recursos eletrônicos para diferentes tarefas está cada vez mais presente na vida cotidiana das pessoas. No Brasil, há cerca de 134 milhões de usuários de internet que utilizam o celular como meio de acesso (TIC Domicílio, 2020), em que as ferramentas mais utilizadas são as redes sociais, correio eletrônico, jogos online e aplicativos de mensagens instantâneas (UNICEF, 2013). As inovações tecnológicas como smartphones, tablets, Ipods, notebooks e televisões tornaram-se acessíveis aos adolescentes e estão disponíveis nos domicílios, bem como o acesso à internet, resultando em muitas horas de acesso (Silva, \& Silva, 2017). Entre as crianças e adolescentes brasileiros, $86 \%$ estão conectados à internet, o que corresponde a 24,3 milhões de usuários (TIC Kids, 2018) e corrobora a expansão do uso desse recurso, especialmente entre os mais jovens.

Embora os meios tecnológicos propiciem comodidade em diversos aspectos, há uma maior sobrecarga e acúmulo de informações. As relações humanas desconectaram-se do mundo real e estreitaram-se no mundo virtual, gerando novos desafios (Costa, Morais, Souza, \& Cabral, 2020). Em 2018, 41\% dos usuários de internet entre 9 e 17 anos relataram contatos com pessoas que não conheciam pessoalmente, com uma proporção maior entre os meninos (44\%) do que entre as meninas (38\%) (TIC Kids, 2018).

À medida que a tecnologia se torna uma parte maior das atividades diárias dos adolescentes, as vantagens e desvantagens das mídias digitais sobre o desenvolvimento psicossocial do adolescente são motivos de preocupação (Shah et al., 2019). Como aspectos positivos desse avanço da disseminação de tecnologia, os adolescentes relatam que a comunicação virtual possibilitou o uso de novas linguagens, o que pode favorecer a liberdade de expressão e o espaço virtual configurou-se como um laboratório social para a vida real, de modo que o acesso à internet pode auxiliar na superação das dificuldades sociais, conflitos emocionais e timidez (Comitê Gestor da Internet no Brasil, 2016). No entanto, comportamentos típicos dos adolescentes, como curiosidade e impulsividade, permitem que ultrapassem alguns limites como os desafios virtuais, selfies em locais extremos ou sexting, um termo que se relaciona à própria exposição, abalando a autoimagem e autoestima (Felt, \& Robb, 2016).

A tecnologia e seus avanços não são inimigos dos adolescentes. Entretanto, por esses encontrar-se em fase especial de desenvolvimento biopsicossocial, deve haver cautela quanto ao acesso, sobretudo quando existe desproporcionalidade no grau de maturidade e discernimento (Tono, 2015). Há necessidade de fornecer a essa população ferramentas para abordar e/ou discernir a relevância e a qualidade de todos os tipos de conteúdo acessados nas mídias digitais (UNICEF, 2020), bem como identificar os obstáculos que pais e cuidadores encontram no controle parental e promover o equilíbrio necessário entre garantir a segurança do adolescente sem a invasão de privacidade (Brasil, 2020).

Em um mundo altamente digitalizado em que muitos jovens aprendem a socializar usando a internet, é de extrema importância identificar e compreender as implicações do uso desses dispositivos, pois o uso inadequado pode resultar em impacto psicológico e comportamental, podendo resultar em perda de controle quanto ao uso da internet, sentimento de culpa, isolamento, sintomas de ansiedade, isolamento, depressão, baixa autoestima, conflito familiar, declínio no desempenho escolar, que são fatores que incidem diretamente na saúde biopsicossocial dos adolescentes, o que enfatiza a necessidade de prevenção (Favotto et al., 2019; Whiteman, 2014). A saúde mental é definida como um estado de bem-estar no qual o indivíduo realiza as suas próprias habilidades, lida com o estresse normal da vida, trabalha produtivamente e frutuosamente, sendo capaz de contribuir com sua comunidade (Organização Mundial da Saúde - OMS, 2014). 
Os jogos eletrônicos e as redes sociais, tornaram-se atividades de lazer para adolescentes e quando o uso se torna bastante intenso, estes jovens podem desenvolver um comportamento de dependência, com impactos na saúde mental. Geralmente esse transtorno resulta na falta de controle do uso da internet, jogos e/ou smartphones, ocasionando sofrimento intenso e/ou prejuízo significativo em diversas áreas da vida, como relacionamentos interpessoais e desempenho nos estudos (Spritzer et al., 2016). Estudos apontam que grande parte dos adolescentes relatam entender os danos à saúde mental causados pelas mídias sociais, principalmente nas situações relacionadas ao cyberbullying e a dependência de smartphones (O'Reilly et al., 2018).

Entre $10 \%$ e $40 \%$ de crianças e adolescentes acessaram ou foram expostos a conteúdos delicados, como suicídio, anorexia, drogas ou violência (UNICEF, 2020). Dessa forma, a tecnologia e as plataformas da internet não devem ser brinquedos oferecidos como forma de distração e entretenimento (Abreu, 2019), especialmente em função de que o uso inadequado das redes sociais, jogos, pornografias apontam sintomas idênticos às outras dependências como fissura, tolerância e abstinência. A dependência da internet pode resultar em transtornos de humor, transtorno de personalidade antissocial e transtornos alimentares (Picon et al., 2015).

Há muita informação disponível na internet, e algumas não são seguras para crianças e adolescentes, o que acarreta a necessidade de cuidados extras em relação ao que é absorvido (Bouzas, 2013). O Estado deve garantir a proteção dos direitos de crianças e adolescentes no ambiente digital. Desde 2019 o Brasil participa de um acordo internacional com a Aliança Global "WePROTECT" no combate à pedofilia e outras formas de abuso de crianças e adolescentes na internet (Brasil, 2020). O percurso mais adequado a se adotar diante dos impactos da tecnologia na saúde física e mental dos adolescentes é a educação digital, com o reconhecimento, esclarecimento e informações sobre os riscos e benefícios advindos do uso das mídias digitais, auxiliando os adolescentes a lidarem e responderem às situações às quais são expostos (Safernet Brasil, 2018).

$\mathrm{O}$ uso indiscriminado das tecnologias nos tempos atuais faz emergir questionamentos em torno do seu impacto. Se por um lado a tecnologia é imprescindível e até mesmo indispensável, por outro traz consequências que requerem atenção, principalmente aos jovens que permanecem imersos no mundo tecnológico. A partir dessas considerações, este estudo buscou identificar os impactos da exposição à internet e mídias sociais na saúde mental dos adolescentes.

\section{Metodologia}

Para alcançar o objetivo proposto neste trabalho, executou-se uma revisão integrativa da literatura seguindo o modelo de Hopia, Latvala e Liimatainen (2016), composto por 4 etapas:

1) Identificação do problema de estudo e revisão de literatura: quais os impactos da exposição à internet e mídias sociais na saúde mental dos adolescentes?

2) Definição dos critérios de inclusão, que são: estudos teóricos e empíricos publicados sem determinação do tempo, disponibilizados nas bases de dados Science Direct, Scopus e BVS. Os descritores utilizados foram: "mental health AND adolescence AND internet AND digital media". Foram selecionados os textos considerando título, resumo e palavras-chave, conforme o DeCS (Descritores em Ciências da Saúde). Identificou-se um total de 245 documentos distribuídos entre as bases de dados: Web of Science (13), Scopus (40), BVS (104) Psycnet (88).

3) Critérios de exclusão para análise dos documentos selecionados: Os textos foram analisados com base em dois filtros sequenciais: a) leitura dos 54 resumos, com retirada dos textos duplicados entre as bases; b) exclusão dos 14 artigos que não contemplavam os aspectos relacionados à exposição à internet e mídia digital e adolescentes e saúde mental

A partir disso, foram incluídos 40 artigos relacionados ao objetivo deste estudo. Além destes textos centrais para este estudo, documentos sobre Saúde Mental foram incluídos, bem como aqueles relacionados à Internet e Mídias Digitais. 


\section{Resultados e Discussão}

A internet passa a ser o palco da busca pela individualidade e acaba gerando uma situação paradoxal: se por um lado é um lugar distante do monitoramento dos pais, por outro torna-se o espaço de exposição que pode atingir graus extremos. Os adolescentes postam dados pessoais, narram detalhes do cotidiano, publicam fotos e vídeos, e em muitos casos tornam público o que é privado, colocando a si mesmo em uma situação de vulnerabilidade. Essas situações expõem os adolescentes a situações de cyberbullying, ansiedade, pesadelos, oscilação de humor, depressão, pensamentos suicidas e suicídio. Constata-se, assim, a vulnerabilidade dos jovens quanto ao acesso às redes sociais.

Como achados nas pesquisas analisadas, identificou-se que os jovens geralmente se adaptam rapidamente às novas tecnologias, potencializando os riscos de impactos à saúde, bem-estar e no desenvolvimento social e econômico. Em todo o mundo, as redes sociais se tornaram um dos principais modos de uso da mídia digital, especialmente para os adolescentes (Weinberg, Ballonoff, \& Dahl, 2021). Do ponto de vista do desenvolvimento humano, é importante reconhecer que as experiências diárias nas quais o aprendizado e o desenvolvimento do cérebro ocorrem acontecem cada vez mais no contexto das plataformas digitais (Giovanelli, Ozer, \& Dahl, 2020).

Com o contexto da pandemia de COVID-19, as interações nos espaços digitais tiveram aumento na frequência e intensidade. As mídias sociais e jogos se configuraram como uma das maneiras de distração, bem como para manter os jovens e suas famílias seguras, em isolamento social, facilitando a comunicação (Granic, Morita, \& Scholten, 2020). Alguns estudos apontam que cerca de $20 \%$ dos adolescentes americanos permanecem mais de dez horas no acesso às telas, fato que pode estar relacionado com o aumento nas doenças psiquiátricas e internações de adolescentes (Mojtabai, Olfson, \& Han, 2016).

O tempo de tela não se limita somente a televisão ou computador, mas também a programas, videogames, mensagens de texto, redes sociais, ouvir música e em diversas atividades que estão vinculadas de alguma forma às telas e a tecnologia. A maioria dessas mídias são sociais, permitindo a comunicação com outras pessoas e o compartilhamento de conteúdo. O acesso não monitorado às redes sociais pode aumentar o risco de bullying, ciberbullying e problemas acadêmicos (Carson, Gansner, \& Khang, 2018).

As mídias digitais são ferramentas importantes e de longo alcance e os adolescentes as usam para desenvolverem suas identidades e receberem feedback de colegas. Essas plataformas podem influenciar na autoestima do adolescente, na imagem e aceitação do próprio corpo. Muitas adolescentes são pressionadas pela sociedade a se adequarem a determinados padrões de beleza e recorrem com mais frequência a softwares de edição de imagens, o que pode aumentar a prática de cyberbullying e transtornos relacionados à imagem corporal (Kelleghan, et al., 2020; Shah et al., 2019). As adolescentes tendem a acessar a internet com mais frequência e a relatar sentimentos de solidão e desconexão social (Coughlan et al., 2021; Lupton \& Deborah, 2021), em que se observa mais comportamentos de comparação com as fotos/imagens das demais colegas e mais relatos de transtornos alimentares (Lonergan et al., 2020) especialmente aquelas com transtornos psiquiátricos devido à baixa autoestima e insegurança nos relacionamentos (Durkee et al., 2012). Alguns estudos apontaram que as adolescentes passam mais tempo nas redes sociais do que os adolescentes do sexo masculino (Lonergan et al., 2019). Em contrapartida, esses últimos, apresentam maior probabilidade de transtornos de dependência dos jogos virtuais, comportamentos antissociais, dificuldade de regulação emocional (raiva), angústia, problemas de autoestima, hiperatividade e desatenção do que as meninas (Wartberg et al., 2017).

Os jovens gastam em média 7 horas por dia nas mídias e a grande maioria tem acesso a uma televisão no quarto, computador, internet, videogame e smartphones. Evidências recentes demonstram os efeitos da mídia sobre a agressividade, comportamento sexual inapropriado, uso de substâncias, transtornos alimentares e dificuldades acadêmicas, bem como relatos de hiperatividade e distração (Männikkö et al., 2020; Strasburger, Jordan, \& Donnerstein, 2010; Lupton, \& Deborah, 2021) e comportamentos de quebrar regras (Männikkö, 2020). Esses elementos podem ser considerados como um fator de risco 
significativo para a automutilação, dificuldades interpessoais no ambiente familiar e baixa motivação na aprendizagem (Wang, Liu, Liu, \& Jia, 2020; Shuai et al., 2021)

Nos Estados Unidos, adolescentes com quadros de epilepsia demonstram se envolver mais em conversas digitais por se sentirem sozinhos e acreditarem que as convulsões afetam os relacionamentos (Falcone et al., 2020). Uma pesquisa feita com um grupo de jovens revelou que cerca de $6 \%$ postam algo sigiloso sobre si de forma anônima nas redes sociais e na grande maioria são questões relacionadas a automutilação, orientação sexual, vivência de bullying escolar, cyberbullying, uso de drogas, sendo os adolescentes do sexo masculino aqueles que participavam de forma mais ativa nessas publicações (Patchin, \& Hinduja 2017). Na Coreia do Sul, devido ao fácil acesso à internet e smartphones, os adolescentes demonstram mau gerenciamento das mídias sociais (Seo et al., 2021). Estudos apontam que uso problemático da internet pode ter relação com o suicídio e cyberbullying (Lissak, 2018; Gansner, 2019) e jovens com deficiências intelectuais e aqueles com histórico de traumas e abusos parecem estar em maior risco de vitimização online, dadas suas vulnerabilidades cognitivas (Norman, 2016; Gansner, Belfort, Leahy, Mirda, \& Carson, 2019).

$\mathrm{Na}$ Itália, os jovens usam a internet em média 12 horas por semana, apresentando dificuldades nas relações interpessoais, nas estratégias de enfrentamento, ansiedade, depressão, retraimento, problemas em seguir regras sociais, transtornos cognitivos e atencionais, agressividade e os adolescentes apresentam maiores índices de vício em jogos (Milani et al., 2018). O uso problemático da internet e o vício em jogos virtuais também ocorrem nos países do sudeste asiático, devido às especificidades culturais e fatores contextuais (Liau et al., 2015). Nos adolescentes da República Tcheca observou-se um uso abusivo da internet com alta prevalência de risco à saúde desses usuários (Dzúrová, Spilková, \& Vraný, 2016). Uma pesquisa realizada com um grupo de jovens, $26 \%$ exibiram uso excessivo de mídia social e $11 \%$ uso excessivo de jogos online, e a idade média de 16 anos com maior probabilidade de uso excessivo das tecnologias, riscos à saúde e consumo de substâncias lícitas e ilícitas (Spilková et al., 2017).

Um estudo realizado na Holanda apontou que 5,7\% de adolescentes relataram dependência a videogames e 9,1\% a uso das redes sociais. Esses dados estão associados a problemas de saúde mental, pensamentos suicidas, hiperatividade, sedentarismo, sendo que os meninos demonstravam maior risco relacionado ao uso de videogames e as meninas associado ao uso das redes sociais (Mérelle et al., 2017). Entre os adolescentes holandeses, 99,8\% usam a internet em casa ou na escola, sendo que $3,7 \%$ destes jovens foram classificados como dependentes da internet. Estes resultados confirmam que a dependência está associada a uma variedade de problemas de saúde física e psicológica e que podem impactar no desenvolvimento dos adolescentes (Kuss et al., 2013). No entanto, também há evidências de que os jogos possuem consequências positivas como prazer, sentimentos de realização, melhora do humor, amizades e um senso de comunidade (Granic et al, 2014).

Diante do aumento do envolvimento dos adolescentes com internet, o transtorno do jogo foi incluído no Manual Diagnóstico e Estatístico de Transtornos Mentais (DSM-5) como uma nova condição que busca compreender e auxiliar por meio de pesquisas clínicas e experiências relacionadas à tecnologia, mais especificamente aos jogos virtuais, incluindo preocupação, tolerância, abstinência (disforia, ansiedade, irritabilidade), perda de controle, uso persistente apesar dos danos com consequências negativas (Griffiths et al., 2016) bem como as tentativas frustradas de controlar o acesso aos jogos, a perda de interesse em antigos passatempos e entretenimentos (American Psychiatric Association, 2013).

Estudos na Finlândia apontam que o uso excessivo da internet causa nos adolescentes exaustão, sintomas depressivos e o sentimento de inadequação. As adolescentes parecem apresentar mais sintomas depressivos (Salmela-Aro et al., 2017; Gansner, 2019). Na Alemanha o uso frequente de computadores, internet e televisão geralmente associa-se a um aumento de problemas emocionais e de relacionamento com os pares e o uso excessivo dos recursos tecnológicos parece relacionar-se à diminuição no bem-estar psicológico (Poulain et al., 2019). Os adolescentes canadenses relataram uso excessivo das mídias 
sociais como instagram, snapchat e facebook, aplicativos e programas que possibilitam a conectividade constante nos relacionamentos, especialmente as adolescentes, que relatam dificuldade na interação com a família, resultando em sentimentos de solidão e impactos nas habilidades de comunicação social (Favotto et al., 2019; Adorjan, \& Ricciardelli, 2021).

Adolescentes do Reino Unido possuem um grande envolvimento em atividades online. Cerca de 99,9\% dos jovens relatam acessar diariamente pelo menos uma forma de tecnologia digital e cerca de $20 \%$ passam mais de 12 horas acessando as redes sociais durante a semana. As adolescentes relataram mais horas de acesso aos smartphones, computadores e assistindo a vídeos, e os adolescentes em jogos de computador (Przybylski, \& Weinstein, 2017). Nesse contexto, os pais reconhecem a importância da prevenção e educação digital, pois colabora no entendimento das consequências negativas das mídias sociais e os jogos para os adolescentes, auxiliando na conscientização da utilização das tecnologias e mídias sociais com moderação (Throuvala et al., 2021). A vivência de conflitos familiares/violência e outros eventos estressantes da vida tem sido associada como fatores de risco para o abuso das mídias na adolescência (Ko et al., 2008). O aumento do tempo destinado ao consumo digital está causando alterações no desenvolvimento de atividade física e comprometendo a capacidade de interagir com outras pessoas na "vida real" (Odgers, Candice, Jensen, \& Michaeline 2020). Portanto, é necessário observar sinais de uso problemático da mídia digital e buscar tratamento precoce quando necessário (Pluhar, Kavanaugh, Levinson, \& Rich, 2019).

Os profissionais que utilizam a tecnologia no atendimento aos adolescentes precisam permanecer atentos aos perigos da internet principalmente aos jovens com histórico de situações traumáticas. A possibilidade de atividades online promove o desenvolvimento saudável e as tecnologias mediadas por computador começaram a influenciar cada vez mais o processo de busca por ajuda e apoio de colegas online em relação à saúde mental, por oferecer caminhos em maior agilidade, os jovens aprendem mais sobre seus corpos de forma anônima e privada (Rafla, Carson, \& DeJonga, 2014; Pretorius, Chambers, \& Coyle, 2019; Lupton, \& Deborah, 2021). Os aplicativos e plataformas (YouTube, Tumblr e Instagram) geralmente atendem melhor às necessidades e mais acessados (Lupton, \& Deborah, 2021). Os profissionais da saúde são incentivados a discutir e auxiliar os adolescentes sobre as questões relacionadas à imagem corporal, autoestima, pornografia e no reconhecimento de que as imagens da mídia são geralmente editadas e aprimoradas, assegurando-os sobre as consequências negativas de um uso indiscriminado desses recursos (Shah et al., 2019; Strasburger, Jordan, \& Donnerstein, 2010).

Um estudo realizado por Weinstein et al. (2021) revela pontos positivos ou benefícios das mídias como conexão e apoio social, conteúdos afetuosos, interesses compartilhados, recursos para a educação sobre saúde mental. Os pontos negativos ou riscos relacionam-se ao estresse, acesso a conteúdos inapropriados, hostilidade e comparações auto degradantes. Os dispositivos digitais são uma fonte valiosa de apoio social e extremamente necessários para desenvolver habilidades para as economias no futuro (Odgers, \& Jensen, 2020). O uso da internet pode resultar em comportamentos de dependência, mas também oferece recursos criativos, experiências pessoais e sociais que proporcionam gratificações (Dhir et al., 2015), oferecendo intervenções para a promoção da saúde mental e bem-estar (Granic, Morita, \& Scholten, 2020). Há relatos de utilidade da mídia social na prevenção do suicídio, permitindo um fórum para o compartilhamento de experiências sem julgamentos (Robinson et al., 2016). O acesso à internet facilita o compartilhamento e a organização de informações para adolescentes envolvidos em grupos ativistas e comunitários. No estudo de Vromen (2007), na Austrália, um site fornece serviços de saúde mental específicos para jovens que incentivam a participação ativa dos adolescentes na comunidade e na prestação de serviços.

As plataformas online também acabam servindo como um espaço para burlar o monitoramento dos pais, desenvolver habilidades de resolução de problemas e tarefas desafiadoras (Uhls et al., 2017). Muitas vezes por não terem afinidade com as redes sociais, os pais apresentam dificuldades em reconhecer o uso e os recursos problemáticos associados a era digital (Lee, 2018). Dessa forma, devem ser incentivados a terem a própria conta de mídia social e serem "amigos" dos filhos adolescentes nesses sites para que possam monitorar regularmente as postagens, os bate-papos, além de manterem conversas sobre 
cyberbullying e os perigos de manter contato com estranhos (Shah et al., 2019). O estudo de Babbage, Jackson e Nixon (2018) apontam que as ferramentas de auxílio para o bem-estar dos adolescentes deveriam ser de fácil acesso semelhantes àquelas de uso diário para fins recreativos e sociais para facilmente direcionar a esse público informações e apoio adequados em saúde mental.

Até o momento, há poucas pesquisas sobre como os pais, profissionais de saúde, escolas, a indústria do entretenimento ou o governo podem proteger crianças e adolescentes dos efeitos nocivos das mídias (Strasburger, Jordan, \& Donnerstein, 2010). Aponta-se a necessidade de avaliar sobre a saúde mental de adolescentes das diferentes origens sociodemográficas e faixas etárias (Lupton, \& Deborah, 2021), bem como compreender os efeitos a longo prazo (Strasburger, Jordan, \& Donnerstein, 2010). Pesquisas futuras podem desenvolver investigações para observar as diferenças nas motivações e racionalizações dos participantes para determinar quais tratamentos são mais adequados (Patchin, \& Hinduja, 2017) em especial na promoção da psicoeducação (Sussman, \& DeJong, 2018) e como exemplo do uso da internet para a melhora da saúde mental existe a terapia cognitivo-comportamental (Topooco et al., 2019). Há, portanto, a necessidade de psicoeducação familiar sobre o uso das mídias digitais. Estudos futuros também devem se concentrar na identificação dos acessos digitais dos adolescentes com diagnósticos psiquiátricos (Gansner, Belfort, Leahy, Mirda, \& Carson, 2019).

\section{Considerações Finais}

Os adolescentes do século XXI não conhecem o mundo sem a internet. O uso dessa tecnologia faz parte do cotidiano desta nova geração e, por ser um fenômeno relativamente novo, ainda não há pesquisas e estudos em grandes proporções sobre os riscos para estes sujeitos que estão em processo de desenvolvimento. É consensual que o mundo das novas tecnologias de informação e comunicação têm influência em todos nós, especialmente nos adolescentes, que tendem a usar mais esses meios de comunicação. Um fato preocupante é que os jovens, por encontrarem-se em processo de desenvolvimento biopsicossocial, demonstram uma certa imaturidade na avaliação dos riscos e benefícios do uso das tecnologias e redes sociais consumindo informações muitas vezes desvinculadas da realidade.

Embora não possam ser apontadas como único fator causal, as mídias sociais podem contribuir substancialmente com estereótipos de comportamentos e o desenvolvimento de hábitos e práticas dos adolescentes. A idade de início e o excesso de tempo nas mídias digitais, pode afetar os hábitos alimentares, sedentarismo, comportamentos violentos ou agressivos, uso ou abuso de substâncias lícitas e ilícitas, depressão, transtornos de imagem corporal, alterações no ciclo sono/vigília, hiperatividade e transtornos de conduta. A internet é um território de oportunidades e proporciona aos adolescentes acesso às informações para estudos e pesquisas escolares, favorece as relações sociais, os aproxima de amigos e familiares e permite conhecer lugares e culturas. Dessa forma, torna-se necessário a educação digital para que informações pessoais como idade, endereço, números de telefone e colégio onde estuda, e hábitos frequentes e compromissos diários, por exemplo, não sejam compartilhados. Sugere-se aos pais e cuidadores a verificação e monitoramento das atividades que os adolescentes realizam nas mídias sociais.

É impossível pensar nas novas gerações separadas dos meios digitais e da internet, no entanto, equilibrar seus efeitos apresenta-se como um grande desafio. Verifica-se necessidade de atuação conjunta e interdisciplinar dos atores que possam promover proteção integral de crianças e adolescentes: escola, pais, responsáveis, comunidade e Estado. A efetivação do direito à saúde no meio virtual deve ser pauta de discussão e criação de protocolos e medidas exclusivas para os adolescentes quanto à prevenção de todas as formas de violência, por meio de uma linguagem clara e compatível com o seu cotidiano. Portanto, é necessário auxiliar no desenvolvimento do protagonismo das ações e medidas que devem ser adotadas. 


\section{Referências}

Abreu, C. N. (2019). O cérebro digital: como o uso constante da internet está afetando nossa mente. https://cristianonabuco.blogosfera.uol.com. br/2019/07/16/o-cerebro-digital-comouso-constante-da-internet-afetando-nossa-mente

Adorjan, M, \& Ricciardelli, R. (2021). Smartphone and social media addiction: Exploring the perceptions and experiences of Canadian teenagers. Canadian Review of Sociology Revue Canadienne de Sociologie, 58(1), 45-64. https://doi.org/10.1111/cars.12319

American Psychiatric Association. (2013). Diagnostic and statistical manual of mental disorders (DSM-5), 5th ed., WashingtonBabbage, C. Jackson, G. M., \& Nixon, E. (2018). Desired Features of a Digital Technology Tool for Self-Management of Well-Being in a Nonclinical Sample of Young People: Qualitative Study. JMIR Ment Health, 18(4), e10067. https://doi.org/10.2196/10067

Bouzas, I. (2013). Internet: um campo de estudo. Adolesc. Saúde, 10(1), 6. https://s3-sa-east1.amazonaws.com/publisher.gn1.co m.br/adolescenciaesaude.com/pdf/v10n1a01.pdf

Brasil (2020). Proteção de crianças e adolescentes na internet. Secretaria nacional dos direitos da criança e do adolescente. Secretaria nacional da família ministério da mulher, da família e dos direitos humanos. Brasília - DF.

Carson, N., Gansner, M., \& Khang, J. (2018). Assessment of Digital Media Use in the Adolescent Psychiatric Evaluation. Child and adolescent psychiatric clinics of North America, 27(2), 133-143. https://doi.org/10.1016/j.chc.2017.11.003

Comitê Gestor da Internet no Brasil. (2019) Núcleo de Informação e Coordenação do Ponto BR. Pesquisa sobre o uso da internet por crianças e adolescentes no Brasil: TIC Kids online Brasil 2018. https://cetic.br/media/docs/publicacoes/216370220191105/tic_kids_online_2018_livro_eletronico.pdf

Comitê Gestor da Internet no Brasil. (2016). Núcleo de Informação e Coordenação do Ponto BR. Pesquisa sobre o uso da internet por crianças e adolescentes no Brasil: TIC Kids online Brasil 2015 http://www.cgi.br/media/docs/publicacoes/2/TIC_Kids_2015_LIVRO_ELETRONICO

Costa, F. M., Morais, M., Souza, C. O., \& Cabral, H. G. (2020). O Repensar das novas tecnologias e a saúde mental na adolescência e juventude: Um desafio para o nosso tempo. Rev. Ambiente Acadêmico,6(1), 84-102. https://multivix.edu.br/wp-content/uploads/2020/07/revista-ambiente-academico-v06-n01artigo05.pdf.

Coughlan, H., et al. (2021). Online Mental Health Animations for Young People: Qualitative Empirical Thematic Analysis and Knowledge Transfer. - $J$ Med Internet Res, 23(2), e21338. https://doi.org/10.2196/21338

Dhir, A., Chen, S., \& Nieminen, M. (2015). Predicting adolescent Internet addiction: The roles of demographics, technology accessibility, unwillingness to communicate and sought Internet gratifications. Computers in Human Behavior, 51, 24-33. https://doi.org/10.1016/j.chb.2015.04.056

Durkee T, et al. (2012). Prevalence of pathological internet use among adolescents in Europe: demographic and social factors. Addiction. 107(12), 2210-22. https://doi.org/10.1111/j.1360-0443.2012.03946.x.

Dzúrová, D., Spilková, J., \& Vraný, M. (2016). Substance misuse and its risk perception in European teenagers. Children's Geographies, 14(2), 203-216. https://doi.org//10.1080/14733285.2015.1028895

Falcone, T. (2020). Digital conversations about suicide among teenagers and adults with epilepsy: A big-data, machine learning analysis. Epilepsia, 61(5), 951-958. https://doi.org/10.1111/epi.16507

Favotto, Lin., Michaelson, V., Pickett, W., \& Davison, C (2019). The role of family and computer-mediated communication in adolescent loneliness. - PLoS One, 14(6), e0214617. https://doi.org//10.1371/journal.pone.0214617

Felt, L. J., \& Robb, M. B. (2016). Technology addiction: concern, controversy, and finding balance.Common Sense Media. https://www.researchgate.net/publication/318707843_Technology_Addiction_Concern_Controversy_and_Finding_Balance.

Fundo das Nações Unidas para a Infância (UNICEF) (2013). O uso da internet por adolescentes. Brasília. »https://www.unicef.org/brazil/pt/br_uso_internet_adolescentes.pdf

Gansner M. (2019). Cyberpsychology, behavior and social networks. The Official Journal of the International Association of CyberPsychology, Training \& Rehabilitation, 349-354. https://phys.org/journals/cyberpsychology-behavior-and-social-networking/

Gansner, M., Belfort, E., Leahy, C., Mirda, D., \& Carson, N. (2019). An Assessment of Digital Media-Related Admissions in Psychiatric Hospitalized Adolescents. Adolescent Psychiatr, 9(3). https://doi.org/10.2174/2210676609666190221152018

Giovanelli, A., Ozer, E. M., \& Dahl, R. E. (2020). Leveraging Technology to Improve Health in Adolescence: A Developmental Science Perspective. The Journal of adolescent health: official publication of the Society for Adolescent Medicine, 67(2S), S7-S13. https://doi.org/10.1016/j.jadohealth.2020.02.020

Granic, I., Lobel, A., \& Engels, R. C. M. E. (2014). The benefits of playing video games. American Psychologist, 69(1), 66-78.

Granic, I., Morita, H., \& Scholten, H. (2020). Young People's Digital Interactions from a Narrative Identity Perspective: Implications for Mental Health and Wellbeing. Psychological Inquiry, 31(3), 258-270. https://doi.org/10.1080/1047840X.2020.1820225

Griffiths, M. D., et al. (2016). Working towards an international consensus on criteria for assessing internet gaming disorder: A critical commentary on Petry et al. (2014). Addiction, 111(1), 167-175. https://doi.org/10.1111/add.13057

Hopia, H., Latvala, E., \& Liimatainen, L. (2016). Reviewing the methodology of an integrative review. Scand J Caring Sci, 30(4), 662-669. https://doi.org/10.1111/scs.12327 
Kelleghan, A. R, et al. (2020). Use of digital media and subsequent initiation of cannabis and tobacco use among adolescents. Drug and alcohol addiction, 212, 108017. https://doi-org.ez46.periodicos.capes.gov.br/10.1016/j.drugalcdep.2020.108017

Ko, C. H., Yen, J. Y., Yen, C. F., Chen, C. S., Weng, C. C., \& Chen, C. C. (2008). The association between internet addiction and problematic alcohol use in adolescents: the problem behavior model. Cyberpsychology, Behavior and Social Networking, 11(5), 571-576. https://doi.org/10.1089/cpb.2007.0199

Kuss, D., Rooij, A.J., Shorter, G., Griffiths, M., \& Mheen, D. V. (2013). Internet addiction in adolescents: Prevalence and risk factors. Comput. Hum. Behav., 29, 1987-1996. https://doi.org/10.1016/j.chb.2013.04.002

Lee, S. Y., Lee, D., Nam, C. R., Kim, D. Y., Park, S., Kwon, J. G., Kweon, Y. S., Lee, Y., Kim, D. J., \& Choi, J. S. (2018). Distinct patterns of Internet and smartphone-related problems among adolescents by gender: Latent class analysis. Journal of behavioral addictions, 7(2), 454-465. https://doi.org/10.1556/2006.7.2018.28

Liau, A. K., Choo, H., Li, D., Gentile, D. A., Sim, T., \& Khoo, A. (2015). Pathological video-gaming among youth: A prospective study examining dynamic protective factors. Addiction Research \& Theory, 23(4), 301-308. https://doi.org/10.3109/16066359.2014.987759

Lissak G. (2018). Adverse physiological and psychological effects of screen time on children and adolescents: Literature review and case study. Environmental research, 164, 149-157. https://doi.org/10.1016/j.envres.2018.01.015

Lonergan, A. R. et al. (2020). Protect me from my selfie: Examining the association between photo-based social media behaviors and self-reported eating disorders in adolescence. Int J Eat Disord. 53:755-766. https://doi.org/10.1002/eat.23256

Lonergan, A. R, Bussey, K., Mond, J., Brown, O., Griffiths, S., Murray, S. B, \& Mitchison, D. (2019). Me, my selfie and me: The relationship between editing and posting selfies and body dissatisfaction in men and women. Body Image, 28, 39-43. https://doi.org/10.1016/j.bodyim.2018.12.001

Lupton, D. (2021). Young People Use of Digital Health Technologies in the Global North: Narrative Review. J Med Internet Res;23(1), e18286. https://doi.org/10.2196/18286

Magis-Weinberg, L., Ballonoff, S, A., \& Dahl, R. E. (2021). Context, Development, and Digital Media: Implications for Very Young Adolescents in LMICs. Frontiers in psychology, 12, 632713. https://doi.org/10.3389/fpsyg.2021.632713

Männikkö N, Ruotsalainen H, Miettunen J, \& Kääriäinen M. (2020) Associations between Childhood and Adolescent Emotional and Behavioral Characteristics and Screen Time of Adolescents. Issues Ment Health Nurs. 41(8), 700-712. https://doi.org/10.1080/01612840.2020.1725195

Mérelle, S. Y. M., Kleiboer, A. M., Schotanus, M., Cluitmans, T. L. M., Waardenburg, C. M., Kramer, D., van de Mheen, D., \& van Rooij, A. J. (2017). Which health-related problems are associated with problematic video-gaming or social media use in adolescents? A large-scale cross-sectional study. Clinical Neuropsychiatry: Journal of TreatmentEvaluation, 14(1), 11-19. https://psycnet.apa.org/record/2017-10293-003

Milani, L., et al. (2018). Internet Gaming Addiction in Adolescence: Risk Factors and Maladjustment Correlates. Int J Ment Health Addiction, 16, 888-904. https://doi.org/10.1007/s11469-017-9750-2

Mojtabai, R., Olfson, M., \& Han, B. (2016). National trends in the prevalence and treatment of depression in adolescents and young adults. Pediatrics, 138 (6), e20161878. http://dx.doi.org/10.1542/peds.2016-1878

Norman (2016). Cyber victim of young people with intellectual or developmental disabilities: specific risks to sexual solicitation J Appl Res Intellect Disabil, 29 (2), 99-110. https://doi.org/10.1111/jar.12163

Odgers, C. L., \& Jensen, M. R. (2020) Annual Research Review: Adolescent mental health in the digital age:facts, fears, and future directions.J Child Psychol Psychiatry, 61(3), 336-348. https://doi.org/10.1111/jcpp.13190

O'Reilly, M., Dogra, N., Whiteman, N., Hughes, J., Eruyar, S., \& Reilly P. (2018). Is social media bad for mental health and well-being? Exploring Adolescents' Perspectives. Clin Child Psychol Psychiatry, 23(4), 601-613. https://doi.org/10.1177/1359104518775154

Patchin, J. W., \& Hinduja, S. (2017). Digital Self-Harm Among Adolescents. The Journal of adolescent health: official publication of the Society for Adolescent Medicine, 61(6), 761-766. https://doi.org/10.1016/j.jadohealth.2017.06.012

Picon, F. et al. (2015). Precisamos falar sobre tecnologia: caracterizando clinicamente os subtipos de dependência de tecnologia. Revista Brasileira de Psicoterapia, 17(2), 44-60. Rhttp://rbp.celg.org.br/detalhe_artigo.asp?id=177.

Poulain, T et al. (2019) Reciprocal Longitudinal Associations Between Adolescente Media Consumption and Psychological Health. Acad Pediatr, 19(1), 109117. https://doi.org/10.1016/j.acap.2018.08.009

Pluhar E, Kavanaugh J.R, Levinson J.A, Rich M. (2019). Problematic use of interactive media in adolescents: comorbidities, assessment, and treatment. Psychol Res Behav Manag, 12, 447-55. https://doi.org/10.2147/PRBM.S208968

Pretorius, C; Chambers, D \& Coyle, D. (2019). Young People Online Help-Seeking and Mental Health Difficulties: Systematic Narrative Review. J Med Internet Res, 21(11), e13873. https://doi.org/10.2196/13873

Przybylski, A. K., \& Weinstein, N. (2017). A large-scale test of the Goldilocks hypothesis: Quantifying the relations between digital-screen use and the mental well-being of adolescents. Psychological Science, 28(2), 204-215. https://doi.org/10.1177/0956797616678438

Radovic, A., McCarty, A., Katzman, K., \& Richardson P. (2018). Adolescents' perspectives on using technology for health: Qualitative study. JMIR Pediatr Parent, 1(1), e2. https://doi.org/10.2196/ pediatrics.8677

Rafla, M., Carson, N. J., \& DeJong, S. M. (2014). Adolescents and the internet: what mental health clinicians need to know. Curr Psychiatry Rep, 16(9), 472. https://doi.org/10.1007/s11920-014-0472-x 
Robinson, J. O, et al. (2016). Social media and suicide prevention: a systematic review. Early Interv Psychiatry, 10, 103-21. https://doi.org/10.1111/eip.12229

Salmela-Aro, K et al., (2017). The Dark Side of Internet Use: Two Longitudinal Studies of Excessive Internet Use, Depressive Symptoms, School Burnout and Engagement Among Finnish Early and Late Adolescents. J Youth Adolesc; 46(2), 343-357 https://doi.org/10.1007/s10964-016-0494-2

Seo, J., Lee, C. S., Lee, Y. J., Bhang, S. Y., \& Lee, D. (2021). The Type of Daily Life Stressors Associated with Social Media Use in Adolescents with Problematic Internet/Smartphone Use. Psychiatry investigation, 18(3), 241-248. https://doi.org/10.30773/pi.2020.0060

Shah, J., Das, P., Muthiah, N., \& Milanaik, R. (2019). New age technology and social media: adolescent psychosocial implications and the need for protective measures. Current opinion in pediatrics, 31(1), 148-156. https://doi.org/10.1097/MOP.0000000000000714

Shuai, L., et al. (2021). Influences of the use of digital media on children and adolescents with ADHD during the COVID-19 pandemic Global Health 17, 48. https://doi.org/10.1186/s12992-021-00699-z

Silva, T. O., \& Silva, L. T. G. (2017). Os impactos sociais, cognitivos e afetivos sobre a geração de adolescentes conectados às tecnologias digitais. Revista Psicopedagogia,34(103). http://pepsic.bvsalud.org/scielo.php?script=sci_arttext\&pid=S0103-84862017000100009

Spilková, J., Chomynová, P., \& Csémy, L. (2017). Predictors of excessive use of social media and excessive online gaming in Czech teenagers. Journal of behavioral addictions, 6(4), 611-619. https://doi.org/10.1556/2006.6.2017.064

Spritzer, D. T. et al. (2016). Dependência de tecnologia: avaliação e diagnóstico. Revista Debates em Psiquiatria. https://studylibpt.com/doc/1475728/depend\%C3\%AAncia-de-tecnologia--avalia\%C3\%A7\%C3\%A3o-ediagn\%C3\%B3stico-artigo-de.

Safernet Brasil (2018). Suposto desafio da "MOMO": recomendações da Safernet Brasil e alerta para pais e educadores. Portal da Safernet Brasil. https://new.safernet.org.br/content/suposto-desafio-da-momo.

Strasburger, V. C., Jordan, A. B., \& Donnerstein, E. (2010) Health effects of media on children and adolescents. Pediatrics, 125(4), 756-67 https://doi.org/10.1542 / peds.2009-2563

Sussman, N., \& DeJong, S. M. (2018). Ethical Considerations for Mental Health Clinicians Working with Adolescents in the Digital Age.Curr Psychiatry Rep;20(12): 113, https://doi.org/10.1007/s11920-018-0974-z

Throuvala, M. A., Griffiths, M. D., Rennoldson, M., \& Kuss, D. J. (2021). Policy Recommendations for Preventing Problematic Internet Use in Schools: A Qualitative Study of Parental Perspectives. International journal of environmental research and public health, 18(9), 4522. https://doi.org/10.3390/ijerph18094522

TIC Domicílios. (2020). Pesquisa sobre o uso de Tecnologias da Informação e Comunicação nos domicílios brasileiros. https://www.cetic.br/media/analises/tic_domicilios_2019_coletiva_imprensa.pdf

Tono, C. C. P. (2015) Análise dos riscos e efeitos nocivos do uso da internet: contribuições para uma política pública de proteção da criança e do adolescente na era digital. Tese (Doutorado em Tecnologia), Universidade Tecnológica Federal do Paraná. Curitiba, 2015. http://repositorio.utfpr.edu.br/jspui/handle/1/1987

Topooco, N. et al., (2019). Evaluating the Efficacy of Internet-Delivered Cognitive Behavioral Therapy Blended With Synchronous Chat Sessions to Treat Adolescent Depression: Randomized Controlled Trial. J Med Internet Res 21(11): e13393 https://doi.org/10.2196/13393

Uhls, Y. T., Ellison, N. B., \& Subrahmanyam, K. (2017). Benefícios e custos das mídias sociais na adolescência. Pediatrics 140 (2), S67-S70. https://doi.org/10.1542/peds.2016-1758E

United Nations Children's Fund - UNICEF (2020). Health and well-being of children and adolescents. Every child has the right to grow up healthy and strong. https://www.unicef.org/health/child-and-adolescent-health-and-well-being

Vromen, A. (2007). Australian young people's participatory practices and internet use. Information, Communication \& Society, 10, 48-68. https://doi.org/10.1080/13691180701193044

Wang L., Liu, X., Liu, Z., \& Jia, C. (2020). Digital media use and subsequent self-harm during a 1-year follow-up of Chinese adolescents. Journal of Affective Disorders, 277, 279-286. https://doi.org/10.1016/j.jad.2020.05.066.

Wartberg, L., Kriston, L., Kramer, M., Schwedler, A., Lincoln, T. M., \& Kammerl R. (2017). Internet gaming disorder in early adolescence: Associations with parental and adolescent mental health. Eur Psychiatry. 43, 14-18. https://doi.org/10.1016/j.eurpsy.2016.12.013

Weinstein, E. et al. (2021). Positive and negative uses of social media among adolescents hospitalized for suicidal behavior. Journal of Adolescence, 87, 6373. https://doi.org/10.1016/j.adolescence.2020.12.003

Whiteman, H. (2014). Facebook may play role in marketing junk food to teens, young adults. Medical News Today. https://www.medicalnewstoday.com/articles/284039.php.

World Health Organization. (2014). Mental health: a state of well-being.: <http://www.who.int/features/factfiles/mental_health/en/ 